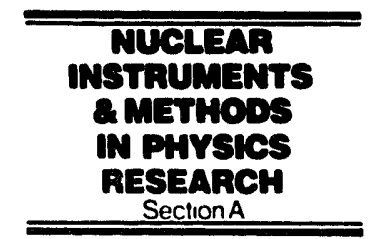

\title{
Mirrors for an imaging gamma ray telescope
}

\author{
Curtis Weaverdyck, Donald Meyer and Carl Akerlof \\ Randall Laboratory of Physics, University of Michigan, Ann Arbor, Michigan 48109-1120, USA
}

Received 14 June 1991

A method is given for making mirrors suitable for large parabolic light collectors. The present application is for collecting Cherenkov light from air showers.

A second $11 \mathrm{~m}$ diameter telescope is being completed on Mt. Hopkins [1] to search for gamma rays from astronomical sources using the Cherenkov light from air showers produced in the atmosphere. The sensitivity for observing such sources is crucially dependent on the ability to reject showers from charged cosmic rays by pattern recognition techniques. The mirror facets must have surfaces accurate to better than one milliradian to optimally accomplish this rejection. Other mirror requirements are that they be reasonable in cost, rugged, have high reflectivity between 330 and $470 \mathrm{~nm}$, and be second surface so that reflectivity does not degrade in the exposed environment encountered in an astronomical setting. To mcet these requirements we adopted a technique pioneered by Argoud [2,3] utilizing aluminized glass bonded to $5 \mathrm{~cm}$ thick glass foam blocks. This article describes how the technique has been modified and improved to attain the desired accuracy. The limitations of this method and the mirror measuring apparatus are also discussed.

The telescope was designed as a parabola with a focal length of $7.6 \mathrm{~m}$. The light gathering surface consists of 433 hexagonally shaped spherical mirror facets of four slightly different curvatures to approximate a parabola which subtends a $38^{\circ}$ cone angle at the focus. The hexagons are $45 \mathrm{~cm}$ across the flats, the size being limited by acceptable astigmatic effects. The mirror glass manufactured by Glaverbel is $0.7 \mathrm{~mm}$ thick to minimize absorption at the shorter wavelengths. The transmission curve is shown in fig. 1. The thin glass with a sagitta of $1.4 \mathrm{~mm}$ conforms accurately and with little pressure to the contour of the glass foam block. The mirrors were aluminized by OCLI of Santa Rosa, CA.

Foamglas, a closed cell foam of pure glass made by Pittsburgh-Corning Corp., is an ideal backing material for mirrors. It is very rigid, has a coefficient of thermal expansion the same as glass, is of low density (5\% that

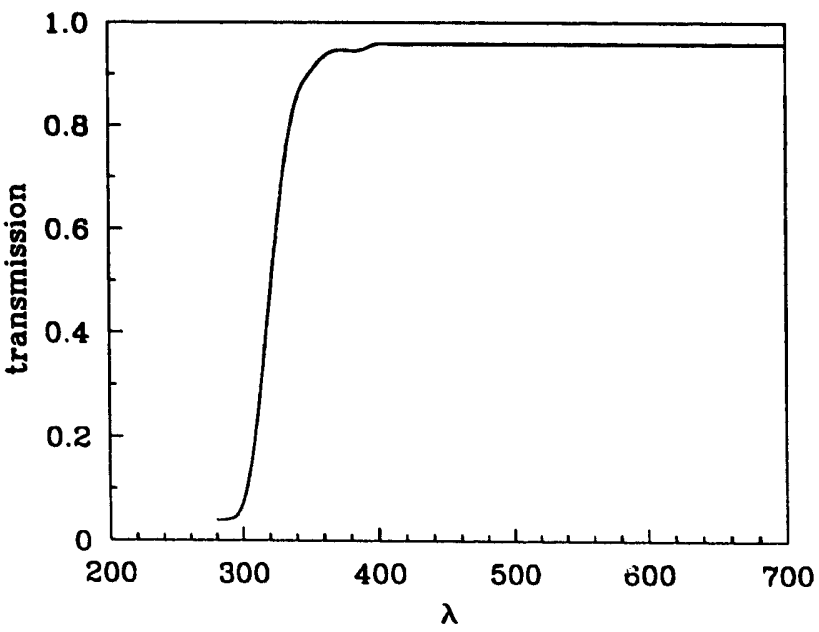

Fig. 1. Transmission of $0.7 \mathrm{~mm}$ mirror glass vs wavelength in $\mathrm{nm}$.

of glass) and is easily worked with bandsaw, knife or machine tool. A form of the desired curvature was made from a $55 \mathrm{~cm}$ diameter, $2.5 \mathrm{~cm}$ thick aluminum jig plate. 180 grit $\mathrm{ZrAl}$ abrasive disks with adhesive backs were applied to the curved surface and the Foamglas blocks were ground by hand on the form to the contour of the plate. The grinding of each block took about $2 \mathrm{~min}$. If care was taken in applying the abrasive disks, the ground surface as measured by the finished mirrors matched the contour of the aluminum to better than $25 \mu \mathrm{m}$. It is clear that some kind of statistical averaging is involved since the dust abraded off is larger than this. One set of abrasive disks was sufficient for making all of the mirrors.

Epoxy (West System 105 resin, 205 hardner) was applied in a thin layer with a foam roller to both the mirror $(10 \mathrm{~g})$ and the Foamglas $(20 \mathrm{~g})$. The Foamglas with the mirror on top, was loaded with another aluminum plate identical with the grinding form. The 


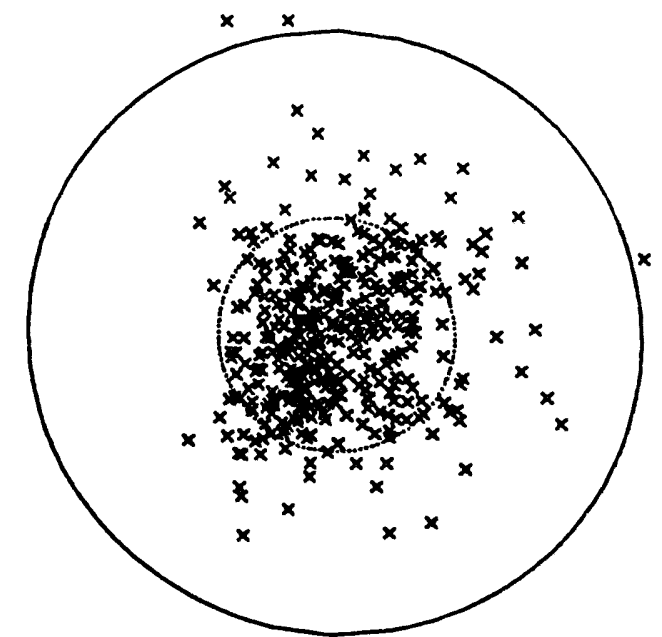

Fig. 2. Distribution of reflected light at the focus of the good mirror as measured on a hexagonal grid. Dashed circle is $1 \mathrm{~cm}$ in diameter.

weight of the aluminum is sufficient to form the mirror (we also tried forming by pulling the mirror down with a vacuum but found this was more tedious and less reliable). The foam makes contact with the mirror over about $1 \%$ of the area. The epoxy flows into the voids in the foam so the mirror surface accurately follows the high points of the shape ground into the foam. The outer $0.5 \mathrm{~cm}$ edge of the mirror crushes the foam slightly in some places. To mask out the resultant flares and protect the glass edge, a bead of black polyurethane caulk is used. The Foamglas is somewhat friable. A covering of Pittcote 404 mastic followed by latex house paint improves durability.
The mirrors are quite rugged. To test for hail damage $1.2 \mathrm{~cm}$ diameter ice cubes were dropped on the surface from a height of $15 \mathrm{~m}$ without causing breakage. $2 \mathrm{~cm}$ diameter cubes sometimes shattered the glass at the point of impact but cracks did not propagate beyond the immediate damage point. A $15 \times 15$ $\mathrm{cm}$ plate containing the mirror mounting adjustments was epoxied to the back of the mirror block. The epoxied joint was strengthened by also epoxying in nine $3 \mathrm{~cm}$ long nails driven into the Foamglas. The assembly was tested in a wind tunnel to $145 \mathrm{~km} / \mathrm{h}$ at various angles without failure. At $170 \mathrm{~km} / \mathrm{h}$ the plate tore off along with cones of Foamglas around each of the nails.

To measure the mirrors a computer controlled optical test facility was constructed to map the curvature of each facet. The basic design consisted of a pair of orthogonal cross slides driven by stepping motors. These were assembled with $45^{\circ}$ mirrors so that a $\mathrm{He}-\mathrm{Ne}$ laser beam could be parallel translated to any point within the $46 \times 53 \mathrm{~cm}$ limiting boundary of a mirror. The coordinates of the reflected beam were measured to approximately $1.5 \mathrm{~mm}$ accuracy by a pair of photodiode sensors mounted to a third motor driven cross slide near the nominal focal point. A $10 \times$ beam expander was used to reduce the laser beam divergence and thus limit the spot size of the reflected light. The entire system was controlled by an IBM. PS/ 2 30-286 microcomputer. On line data analysis produced an immediate display of several statistical $m$ zasures of the mirror surface accuracy. The data was also archived on disk files for further analysis. The results for a good mirror are shown in fig. 2 for a hexagonal grid containing 397 points. The solid circle in the diagram is $2.5 \mathrm{~cm}$

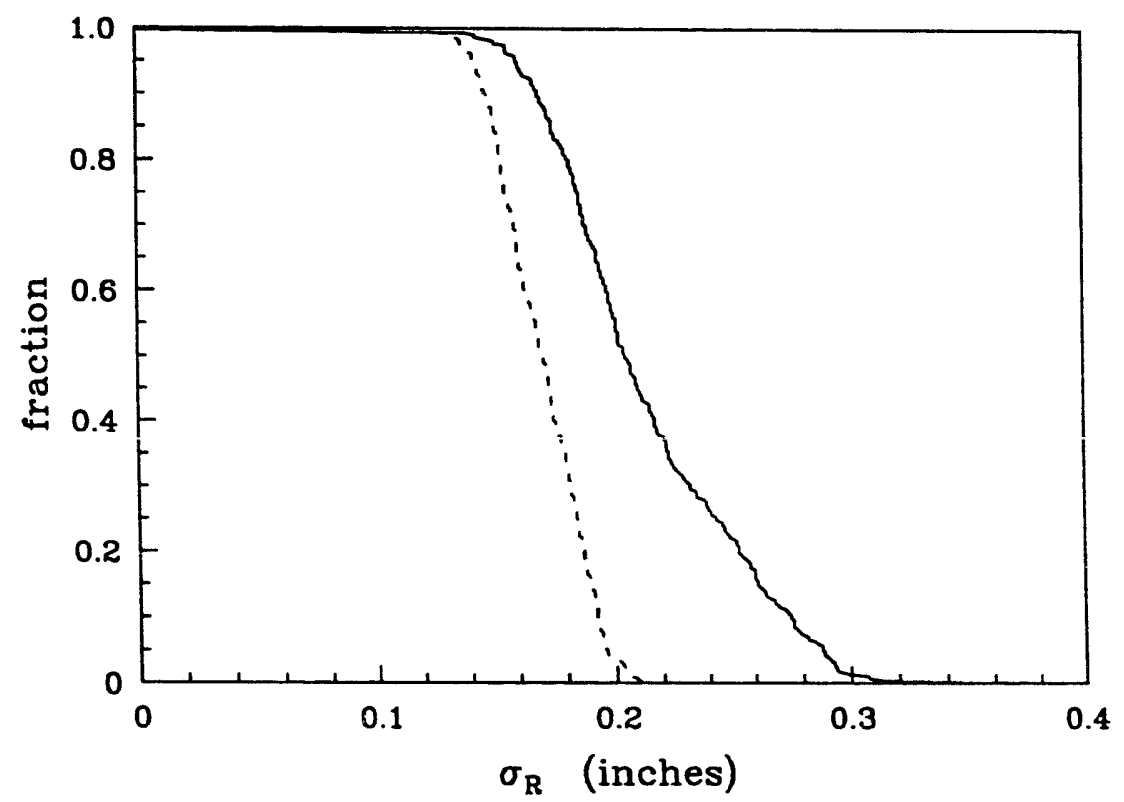

Fig. 3. Ensemble of circular probable errors for 400 mirrors. Solid curve is for unmasked mirrors. dashed for masked. 


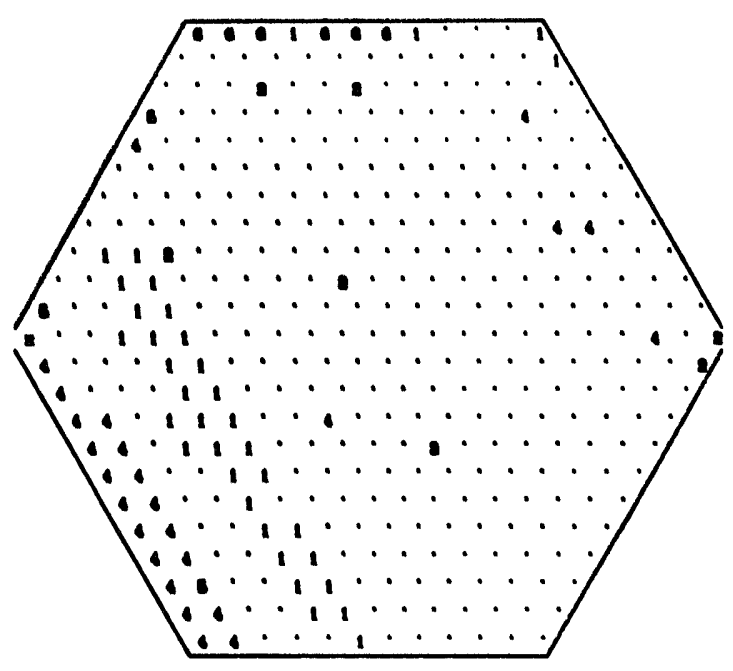

Fig. 4. Mirror with glass thickness striation. Pattern of striation on mirror is clearly visible at left. Effect at focus can be seen at right. Dashed circle $2.4 \mathrm{~cm}$ in diameter. in diameter. The dashed circle containing $80 \%$ of the points is $1 \mathrm{~cm}$, in diameter. In the absence of glass defects, discussed in the next paragraph, the distribution in Gaussian. Fig. 3 shows the ensemble of circular probable errors for 400 mirrors. As orginally made, half of the mirrors have rms errors corresponding to less than $0.3 \mathrm{mrad}$. By masking a small fraction of the area, the rms error can be reduced so that all mirrors satisfy this tolerance. The mirrors meet our criteria and do not contribute significantly to the blurring of the Cherenkov light image of the showers.

How much can the technique be improved? The aluminum forms were accurate to $\pm 0.5 \mathrm{mrad}$ and could be improved with some effort. However the major limitation we encountered was nonuniformities of the order of 50 to $100 \mu \mathrm{m}$ in mirror glass thickness. Typically $35 \%$ of the glass pieces had thickness striations from the drawing process which ran across the glass resulting in optical distortions. A typical mirror pattern caused by a striation is shown in fig. 4 . Other less obvious variations were also present. The glass nonuniformity is dominant enough that it is not possi- ble to tell where other limitations would arise if more uniform glass were available. While requiring some care mirrors can be made relatively inexpensively. Two thirds of the material cost was the mirror aluminization.

\section{Acknowledgements}

The authors wish to thank the U.S. Department of Energy for their support under contract DE-AC0276ER01112.

\section{References}

[1] C.W. Akerlof, et al., Proc. SPIE 33rd Annu. Int. Symp. vol. 1159 (1989) p. 270.

[2] M.J. Argoud, Jet Propulsion Lab. Pub. 900-735 (1976).

[3] M.J. Argoud, Proc. 1 Semi-annual Distributed Receiver Systems Program Rev. Report DoE/JPL-1060-33 (1980) p. 41. 